\title{
SIFT Algorithm For Image Stitching
}

\author{
ZhangPan ${ }^{1, a^{*},}$ RuanJun ${ }^{2, b}$ \\ 1.2.Center for Hydrogeology and Environmental Geology Survey,CGS, Baoding 710000,China \\ a zhangpan@chegs.cn, b ruanjun@chegs.cn
}

Key words: feature points;SIFT descriptor;image registration;image stitching

Abstract. An algorithm based on SIFT feature detection method(Scale Invariant and Feature Transform) and using conversion step of the circular area selection feature points described are introduced into image feature detecting and matching for better real time performance and higher precision.Improved SIFT features 64-dimensional descriptors and SIFT features 88-dimensional descriptor their shortcomings. And the algorithm is applied to image stitching process improved. Experimental verification of the improved method is improved by the time complexity.

\section{Introduction}

Feature extraction from the image registration and mosaic are key role in the process, SIFT algorithm is a method for target identification which is David G. Lowe first proposed in 1999.First, detection between the two images and gray scale space Identify the key position and the scale, and then use the feature point of the neighborhood gradient's main direction as the SIFT direction ,then to match use the key features, but the algorithm still exists mismatch.Then, Based on the feature point invariance and selected areas of the image registration and mosaic method have been widely used and has been improved to reduce the number of feature descriptor vector dimension, improve the matching efficiency has become a hot topic in recent years. Document proposed a SIFT algorithm combined with Canny operator to carry out 96-dimensional feature point matching, its dimension remains high.

\section{SIFT feature descriptor}

Generating scale space and detecting extreme points:

Two-dimensional image of a scale space of the image may be obtained with a Gaussian convolution kernel,namely:

$$
\begin{aligned}
& L(x, y, \sigma)=G(x, y) * I(x, y) \\
& G(x, y, \sigma)=\frac{1}{2 \pi \sigma^{2}}=\exp \left(-\frac{\left(x^{2}+y^{2}\right)}{2 \sigma^{2}}\right)
\end{aligned}
$$

On the basis of the scale space, use scale image difference between adjacent, difference of Gaussian scale space establish that operator:

$$
\begin{aligned}
& D(x, y, \sigma)=(G(x, y, k \sigma)-G(x, y, \sigma)) * \\
& I(x, y)=L(x, y, k \sigma)-L(x, y, \sigma)
\end{aligned}
$$

Feature point feature orientation:

The main direction of the feature point can be made at this point not because of image scaling and rotation and degeneration. Modulus and direction feature point neighborhood pixel gradients are important factors in ensuring the rotation invariant feature point descriptor is calculated at the point where the gradient modulus characteristics as formula (4), the main direction of the feature point is calculated as formula formula (5)

$$
m(x, y)=\sqrt{(L(x+1, y)-L(x-1, y))^{2}+(L(x, y+1)-L(x, y+1))^{2}}
$$


$\theta(x, y)=\arctan \left[\frac{L(x, y+1)-L(x, y-1)}{L(x+1, y)-L(x-1, y)}\right]$

Generating feature point descriptor:

As shown in Figure 1, where each small square represents a pixel, for each feature point are the feature point as the center, select the region $16 \times 16$ around the feature point, then this area were divided into $4 \times 4$ sub-regions, calculate the main direction of 16 pixels for each sub-region, Pixels for a total calculated as $S=16 \times 16$, while access to the $4 \times 4 \times 8=128$ dimensional vectors, denoted SIFT128.
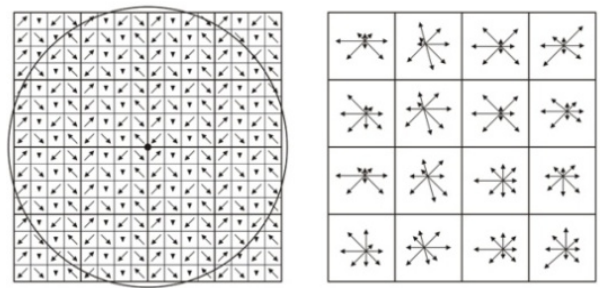

Figure 1 SIFT feature descriptor vector diagram showing

\section{SIFT64}

Tang CM, who used the ring to replace the rectangular region SIFT region, in order to reduce the time to calculate the main direction SIFT generated consumption, thereby reducing the time complexity, which uses fixed step size of one pixel of the feature pointIt will be described, as shown in Figure 2. Take eight sub-rings, each pixel has eight sub-ring feature vector, so the $8 \times 8=64$ dimension feature vector is defined for the feature point descriptor, herein referred to as SIFT64. Area outermost ring is $16 \times 16$ pixels square area, the total amount of the calculated pixel $S_{8}<S<16 \times 16 \quad\left(S_{8}\right.$ for the total number of pixels radius circular region 8 pixels).

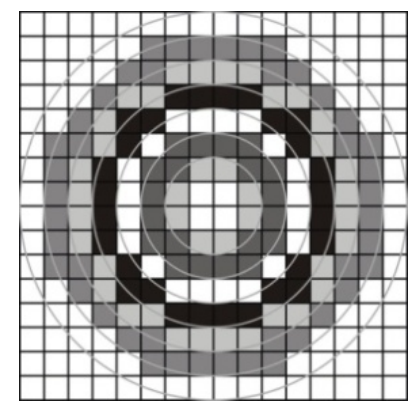

Figure 2 is based on SIFT feature descriptor SIFT64 schematic

\section{SIFT88}

Wu Jian and others on the basis of SIFT64 Tang were proposed to improve the variable step method for calculating the feature descriptor, as shown in Figure 3, it still uses 8 ring that is $(4,4,3,3,2,2,1,1)$ the ring number of feature points are described, but it increases the cumulative value of the difference between positive and negative gray and gray accumulated value on the basis of eight feature vectors of each of the ring, the total dimension number $(8+2+1) \times 8=88$, the paper referred to as SIFT88. Area outermost ring is $40 \times 40$ a square pixel region, the calculated amount of $S_{20}<S<40 \times 40$ pixels. 


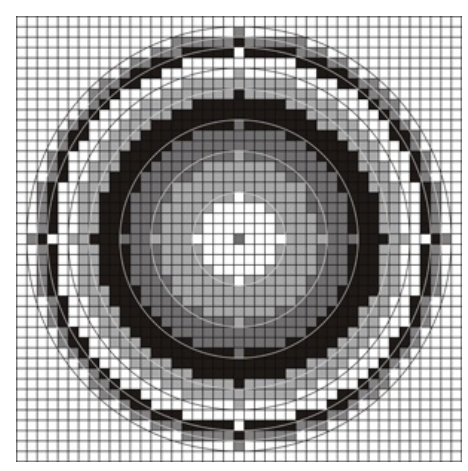

Figure 3 is based on SIFT feature descriptor SIFT88 schematic

In summary, the two algorithms SIFT64 traditional SIFT128 dimension reduced from 128 to 64 dimensions, reduced time complexity; SIFT88 although the SIFT64 to improve, but there are still less than the following:

(1) Used SIFT88 variable step algorithm for sampling, sample offset solve discrete pixels, but too large to calculate pixel whose value is far greater than the S value S SIFT64 of. Lead to high time complexity of the problem, this article will use another algorithm to solve this problem. Greatly reduce the amount of S is calculated. Reduces the time complexity.

(2) SIFT88 algorithm uses the minimum shift method to resolve mismatches, but in practice not only mismatch, there are duplicate matching algorithm which uses not remove duplicate match. This paper method and the exhaustive search and utilizing the rotation invariant feature circular region to simultaneously remove duplicate matching and mismatching problem, to ensure accuracy.

\section{Improved feature descriptor}

For traditional SIFT algorithm, the problems betweenSIFT64 and SIFT88, this paper presents an improved method better:

(1) As shown in Figure4, in this paper, the number of rings instead of converting fixed number of rings SIFT64, namely the sub-loop step is not fixed, but decreases the step size, the closer to the central area, the larger step. Increased sub-pixel weights to minimize mismatch. However, due to an increase in the number of rings will increase the time overhead, so use paper converting step on the basis of the total number of rings has been controlled to reduce the time complexity, from close to the center pixel counting were $(3,3,2,2,1,1)$ the number of rings, the ring so that the total number of ring 12, to calculate the amount of pixel radius of a circular area $3+3+2+2+1+1=12$, provided the total number of pixels is $\mathrm{S}$, then $S_{12}<S<24 \times 24$.

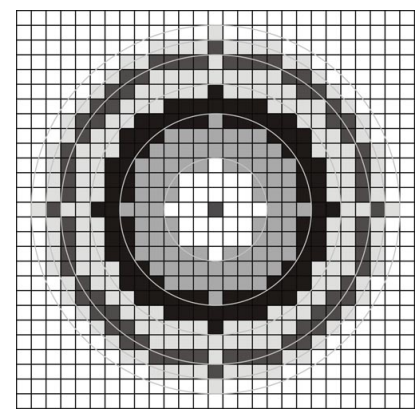

Figure 4 The improved feature descriptor ring configuration diagram

(2) Add a weighting factor ( weight $_{1}$, weight $_{2}$, weight $_{3}$, weight $_{4}$, weight $_{5}$, weight ${ }_{6}$ ), respectively

Control $(3,3,2,2,1,1)$ the degree of influence on the center pixel of each layer. Making closer to the center, the greater the degree of influence, the further away from the center point, the smaller the degree of influence.

(3) With variable sliding window method accumulate scale gradation difference. Ring as a whole is about a large area $\mathrm{R}$, each ring produces a positive and a negative gradation accumulated value of the cumulative 
gradation value $\left(H_{R i+}, H_{R i-}\right)$, when the accumulated value is calculated using the size of the two rings sliding window accumulates, i.e. gray cumulative value of 2 rings first ring and the second ring of the gray scale and the accumulated value of; gray gray cumulative value of the third ring of the second ring and the third ring of the accumulated value sum, and so on . Calculate the cumulative value of each ring of gray, so each ring is 2-dimensional vector descriptor.

(4) Using the original image in the traditional SIFT algorithm to construct a grayscale image Difference of Gaussian pyramid, to improve the matching accuracy, grayscale paper will ring the accumulated value of each sub-gray as a one-dimensional feature vector. gray is calculated by the following formula (6).

gray $=\sum I_{R_{i}}\left(I \in R_{i}\right)(6)$

In the formula, $I$ is he gray value, $R_{i}$ is each annular zone.

(5) Finally, in order to avoid the influence of light generated feature vectors herein do normalized. The improved characteristics herein descriptor consists of three parts: The first part is characterization eight directions symbol cumulative weighted gradient magnitude and direction $(0,45,90,135,180,225,270,315)$ on; the second part is the difference between positive and negative gray cumulative histogram $\left(H_{R i+}, H_{R i-}\right)$; the third part is gray ring around the feature points of the cumulative sum.

To sum up: the local dimension of each descriptor ring is: $8+2+1=11$. The total dimension is $11 \times 6=66$, herein referred to as SIFT 66. Finally, the image is automatically splicing.

\section{Summary}

The SIFT improved algorithm applied to the image stitching, image feature extraction stage dimension dimension reduced from 128 to 66 dimensions to improve the operating speed, and keep the SIFT8 8 stability and accuracy. Based on the splicing process is not treated in more detail, there are still subtle splicing gap, as a follow-up study.

\section{Reference}

[1]Lowe D G. Object recognition from local scale-invariant features[C]//Proceedings of the International Conference on Computer vision.Corfu,1999:1150-1157.

[2]Lowe D G.Distinctive Image Features from Scale-Invariant Keypoints. [J].International Journal of Computer vision,2004,60(2):91-110.

[3] Chunming Tang.Automatic Registration based on Improved SIFT for Medical Microscopic Sequence Images[J]. Second International Symposium on Intelligent Information Technology

[4]Chalechale A,Naghdy G,M ertins A.Sketch based image matching using angular partitioning systems[J].IEEE Trans on Man and Cybernetics,Part A,2005,35(1):28-41. 